\title{
Bone marrow histomorphological criteria can accurately diagnose hemophagocytic lymphohistiocytosis
}

\author{
Eric Gars, ${ }^{1}$ Natasha Purington, ${ }^{1}$ Gregory Scott, ${ }^{1}$ Karen Chisholm, ${ }^{2}$ \\ Dita Gratzinger, ${ }^{1}$ Beth A. Martin ${ }^{1 *}$ and Robert S. Ohgami ${ }^{1 *}$ \\ ${ }^{1}$ Stanford University, CA and ${ }^{2}$ Seattle Children's Hospital and University \\ of Washington, WA, USA \\ $B A M$ and RSO contributed equally to this work.
}

$\mathrm{H}$ emophagocytic lymphohistiocytosis (HLH) is a rare multi-system inflammatory disorder with diagnostic criteria based on the HLH2004 trial. Hemophagocytosis is the only histomorphological criterion, but in isolation is neither specific nor sensitive for the diagnosis of HLH. While objective thresholds for clinical and laboratory criteria have been established, specific criteria for histomorphological evidence of hemophagocytosis in HLH have not been rigorously evaluated or established. We sought to determine if numerical and objective criteria for morphological hemophagocytosis could be identified, and if such criteria would aid in the diagnosis of HLH. We analyzed the morphological features of hemophagocytosis in 78 patients presenting with clinical features suspicious for HLH: 40 patients with and 38 patients without $\mathrm{HLH}$. We demonstrate that non-nucleated erythrophagocytosis alone is a non-specific finding, while hemophagocytosis of granulocytes [1 per 1000 cells, area under the curve (AUC): 0.92, 95\% Confidence Interval (CI): 0.86, 0.99], nucleated erythrocytes (4 per 1000 cells, AUC: 0.92, $95 \%$ CI: $0.87,0.98)$, and at least one hemophagocyte containing multiple nucleated cells (AUC: $0.91,95 \% \mathrm{CI}: 0.85,0.95$ ) are strongly associated with HLH. Joint modeling of hemophagocytes containing engulfed granulocytes, nucleated erythrocytes, and lymphocytes effectively distinguished between HLH and non-HLH (cross-validated AUC: 0.90, $95 \%$ CI: $0.83,0.97)$.

\section{Introduction}

Hemophagocytic lymphohistiocytosis $(\mathrm{HLH})$ is a rare life-threatening syndrome that occurs secondary to severe systemic immune activation. ${ }^{1}$ Cytotoxic T-cell proliferation leads to increased cytokine production and activation of tissue resident macrophages. Ultimately, multi-system end organ damage caused by massive inflammation may lead to a fatal outcome without timely diagnosis and initiation of appropriate therapy.

Hemophagocytic lymphohistiocytosis affects patients of all ages and occurs as an inherited disease, or secondarily in the setting of predisposing conditions that alter the normal immune response. The inherited form of the disease presents in early childhood and is associated with homozygous mutations in genes involved in CD8 ${ }^{+}$T-cell- and NK-cell-mediated immunity. ${ }^{3}$ These genetic forms of HLH are uniformly fatal without hematopoietic cell transplant or gene therapy. Secondary HLH may occur sporadically in healthy individuals, but is more often encountered in patients with hematologic malignancy, autoimmune disease, and iatrogenic immunosuppression. Virtually all cases are thought to require an infectious or noninfectious trigger to initiate the aberrant immune response, regardless of the underlying immune dysfunction. ${ }^{4.7}$

Hemophagocytic lymphohistiocytosis presents abruptly over a period of several days to weeks with a consistent pattern of fever, pancytopenia, and splenomegaly. Common laboratory abnormalities include hyperferritinemia, hypofibrinogenemia, hypertriglyceridemia, elevated soluble IL-2 receptor, and abnormal liver func-
Haematologica 2018

Volume 103(10):1635-1641

\section{Correspondence:}

ericgars@stanford.edu or rohgami@stanford.edu

Received: December 17, 2017. Accepted: June 13, 2018.

Pre-published: June 14, 2018.

doi:10.3324/haematol.2017.186627

Check the online version for the most updated information on this article, online supplements, and information on authorship \& disclosures: www.haematologica.org/content/103/10/1635

(C)2018 Ferrata Storti Foundation

Material published in Haematologica is covered by copyright. All rights are reserved to the Ferrata Storti Foundation. Use of published material is allowed under the following terms and conditions:

https://creativecommons.org/licenses/by-nc/4.0/legalcode. Copies of published material are allowed for personal or internal use. Sharing published material for non-commercial purposes is subject to the following conditions: https://creativecommons.org/licenses/by-nc/4.0/legalcode, sect. 3. Reproducing and sharing published material for commercial purposes is not allowed without permission in writing from the publisher. 

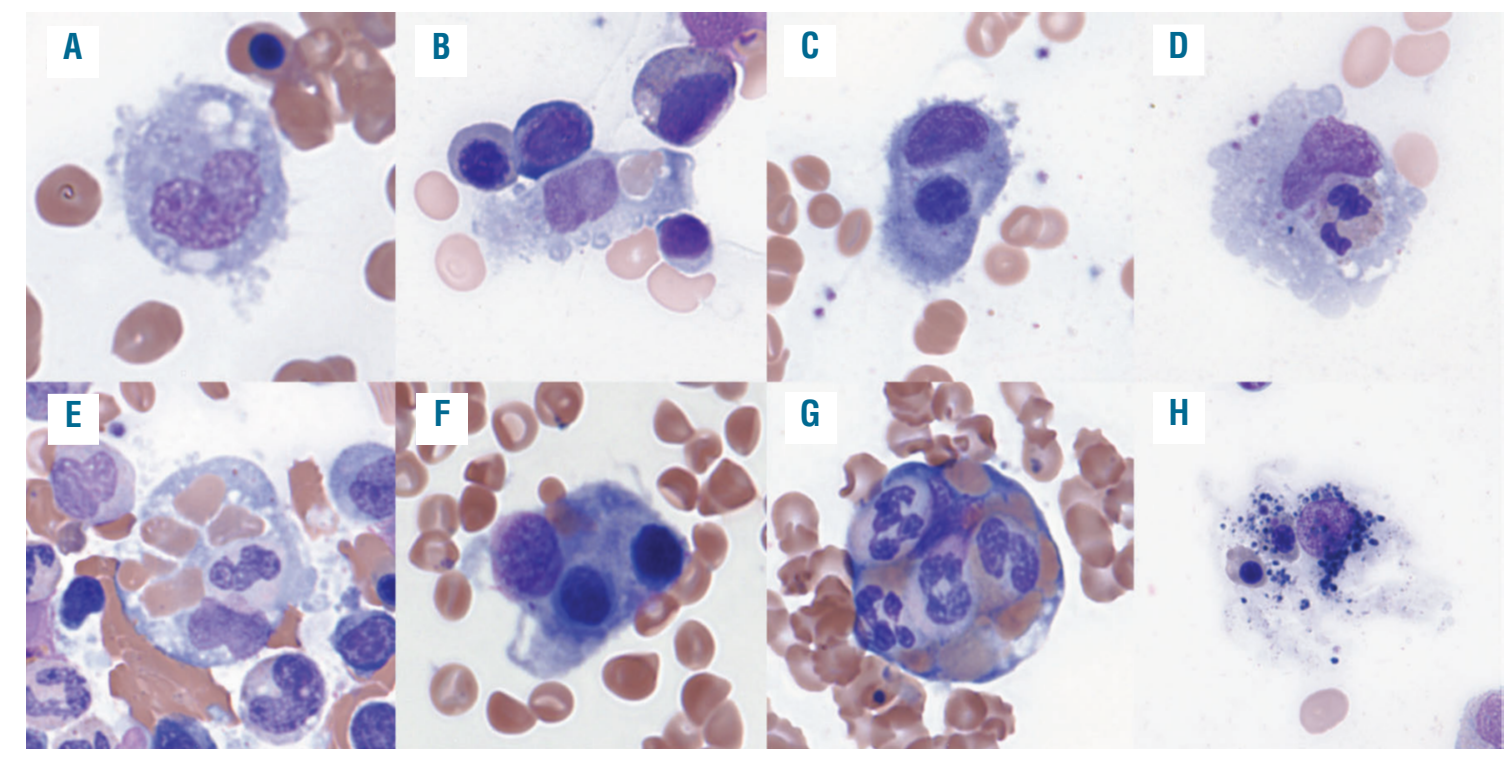

Figure 1. Examples of hemophagocytosis in patients with hemophagocytic lymphohistiocytosis (HLH). (A) Histiocytes in patients with HLH often display rounded contour with cytoplasmic projections. (B-D) Hemophagocytes with a single ingested mature red blood cell (RBC), nucleated RBC progenitor, and granulocyte, respectively. Hematopoietic progenitor cells (HPCs) often contain single nucleated hematopoietic cells in addition to multiple mature RBCs (E); however, the presence of multiple nucleated cells within the cytoplasm of a single HPC ( $F$ and $G)$ is highly predictive of the diagnosis of HLH. (H) An example of a histiocyte with degenerating nuclear debris, indistinct cytoplasmic contour, and equivocal intracytoplasmic nucleated RBCs that we do not consider to be a definite hemophagocyte.

tion tests. ${ }^{1}$ The most widely used diagnostic criteria for HLH were developed for inclusion in the HLH-2004 trial which requires genetic evidence of a mutation associated with HLH or fulfillment of 5 of 8 clinical criteria including fever, splenomegaly, bicytopenia, hypertriglyceridemia or hypofibrinogenemia, evidence of hemophagocytosis in bone marrow or other tissues, low or absent NK-cell activity, elevated ferritin, and elevated soluble IL- 2 receptor. ${ }^{3}$ Although not validated for adults, these HLH-2004 criteria are broadly applied in patients of all ages.

Pathologists play a critical role in the diagnostic workup of patients suspected of having HLH. Bone marrow examination is performed to evaluate for hemophagocytosis, identify underlying malignancy, and exclude benign or neoplastic mimics. The presence of hemophagocytosis in the marrow fulfills one of the HLH-2004 diagnostic criteria; however, no accepted diagnostic threshold or reporting guidelines have been established. The lack of evidence-based guidelines leads to considerable uncertainty among pathologists as to what degree of hemophagocytosis is sufficient to satisfy this criterion. Adding to the challenge is that hemophagocytosis is not specific to the diagnosis of HLH in the absence of other clinical features of the disease. Rare erythrophagocytosis is commonly seen in bone marrow aspirates and increased hemophagocytosis may be encountered in the setting of sepsis, blood transfusions, hematopoietic transplantation, chemotherapy, and myelodysplastic syndrome. . $^{-11}$

Given the lack of a defined threshold to fulfill the criterion for diagnosis of HLH, we designed this retrospective study to interrogate whether quantitative or qualitative morphological features of hemophagocytosis in bone marrow aspirates are predictive of the eventual diagnosis of HLH. We identified a cohort of patients presenting with clinical characteristics that were of concern for $\mathrm{HLH}$ and their aspirates were examined blindly.

Hemophagocytes were enumerated per 1000 nucleated cells according to the lineage of their ingested hematopoietic contents [mature red blood cells (RBCs), nucleated RBCs (nRBCs), granulocytes, and lymphocytes] (Figure 1). In addition to quantitative features, we evaluated a binary morphological feature, the presence of multiple nucleated cells within a single hemophagocyte, as a possible predictive characteristic of HLH.

\section{Methods}

\section{Patient selection}

We searched the pathology Laboratory Information Service database (Powerpath) using the following keywords: "hemophagocytic lymphohistiocytosis", "hemophagocytosis", "erythrophagocytosis", and "HLH". This search returned 258 results between the dates $1^{\text {st }}$ January 2013 and $7^{\text {th }}$ January 2017, and included text from anywhere within the diagnostic report including the provided clinical information, microscopic description, diagnostic line, and/or diagnostic comment (Figure 2). These patients' medical records were reviewed by EG to assess whether clinical suspicion for $\mathrm{HLH}$ was present at the time of bone marrow aspiration, either indicated on the specimen requisition form (i.e. "rule out HLH" or "concern for $\mathrm{HLH}^{\prime \prime}$ ) or listed in the differential diagnosis in the electronic medical record (EMR) within one week prior to biopsy. Demographic information, clinical characteristics, diagnostic impressions, pathological features, and laboratory values at the time of biopsy were collected for each patient. Patients were classified as "HLH" and "non-HLH" based on the diagnostic impression of the consulting hematologists described in the clinical notes. The final diagnosis in all cases was determined based on the HLH-2004 criteria in conjunction with the overall clinical picture. Patients were excluded from the analysis if hemophagocytosis was incidentally noted independent of clinical concern for HLH, slides were not available for review, HLH was considered but the diagnosis was equivocal after workup, or a documented history of HLH-directed treatment was noted prior to biopsy. This study was approved by Stanford University's institutional review board. 
Table 1. Clinical and laboratory findings of patients in hemophagocytic lymphohistiocytosis (HLH) and non-HLH groups.

\begin{tabular}{|c|c|c|}
\hline Patients' characteristics & $\begin{array}{c}\text { 개내 } \\
(N=4,0)\end{array}$ & $\begin{array}{c}\text { Non-HLY } \\
(\mathrm{N}=38)\end{array}$ \\
\hline Age in years, mean (range) & $50(10 \mathrm{mo}-96)$ & $41(1 \mathrm{mo}-85)$ \\
\hline Male, $\%$ & $54 \%$ & $55 \%$ \\
\hline \multicolumn{3}{|l|}{ HLH-2004 Criteria ${ }^{\dagger}$} \\
\hline Fever $\geq 38.5^{\circ} \mathrm{C}, \mathrm{n} / \mathrm{N}(\%)^{\dagger}$ & $38 / 40(95 \%)$ & $29 / 38(76 \%)^{*}$ \\
\hline Splenomegaly, $\mathrm{n} / \mathrm{N}(\%)^{\dagger}$ & 26/37 (70\%) & $16 / 32(42 \%)^{*}$ \\
\hline Bicytopenia, $\mathrm{n} / \mathrm{N}(\%)^{\dagger}$ & $38 / 40(95 \%)$ & $22 / 38(58 \%)^{*}$ \\
\hline Triglycerides $>265$ mg/dL, n/N $(\%)^{\dagger}$ & $15 / 38(39 \%)$ & $2 / 31(6 \%)^{*}$ \\
\hline Triglycerides, mean (range), mg/dL & $301(49-812)$ & $174(39-479)^{*}$ \\
\hline Fibrinogen $<150 \mathrm{mg} / \mathrm{dL}, \mathrm{n} / \mathrm{N}(\%)^{\dagger}$ & $17 / 39(44 \%)$ & $3 / 33(9 \%)^{*}$ \\
\hline Fibrinogen, mean (range) & $262(57-779)$ & $413(64-1200)^{*}$ \\
\hline Ferritin $>500 \mathrm{ng} / \mathrm{mL}, \mathrm{n} / \mathrm{N}(\%)^{\dagger}$ & $39 / 40(98 \%)$ & $27 / 36(75 \%)^{*}$ \\
\hline Ferritin, median (range), ng/mL & $19,331(425-40000)$ & $6,553(30-40,000)^{*}$ \\
\hline Elevated soluble IL-2 receptor ( $>2400 \mathrm{U} / \mathrm{mL}), \mathrm{n} / \mathrm{N}(\%)^{\dagger}$ & $30 / 32(94 \%)$ & $7 / 21(33 \%)^{*}$ \\
\hline Soluble IL-2 receptor, median (range), U/mL & $14,963(1215-69,000)$ & $1782(139-4,431)^{*}$ \\
\hline Decreased NK-cell function, $\mathrm{n} / \mathrm{N}(\%)^{\dagger}$ & 2/12 (17\%) & $3 / 8(38 \%)^{*}$ \\
\hline Hemophagocytosis, n/N (\%) ${ }^{\dagger}$ & $40 / 40(100 \%)$ & $12 / 38(32 \%)^{*}$ \\
\hline Malignancy, n/N (\%) & $22 / 40(56 \%)$ & $9 / 38(24 \%)^{*}$ \\
\hline Autoimmune disease, n/N (\%) & $11 / 40(28 \%)$ & 15/38 (38\%) \\
\hline \multicolumn{3}{|l|}{$\begin{array}{l}\text { Hemophagocytosis Criteria Sum } \\
\text { (excluding hemophagocytosis), n/N (\%) }\end{array}$} \\
\hline 0 & 0 & $1(3 \%)$ \\
\hline 1 & 0 & $4(11 \%)$ \\
\hline 2 & 0 & $7(18 \%)$ \\
\hline 3 & $2(5 \%)$ & $14(37 \%)$ \\
\hline 4 & $9(23 \%)$ & $8(21 \%)$ \\
\hline 5 & $16(40 \%)$ & $4(11 \%)$ \\
\hline 6 & $12(30 \%)$ & 0 \\
\hline 7 & $1(3 \%)$ & 0 \\
\hline
\end{tabular}

n/N: number; mo: months; * $P<0.05$. 'One of HLH-2004 criteria (one criteria is fibrinogen $<150 \mathrm{mg} / \mathrm{dL}$ or triglycerides $>265 \mathrm{mg} / \mathrm{dL}$ ).

\section{Evaluation of bone marrow aspirates}

Bone marrow aspirates (Wright-Giemsa stained) of $\mathrm{HLH}$ and non-HLH patients were evaluated blindly. Every aspirate slide was initially examined at low power (4x) to identify areas with hemophagocytosis and select an appropriate slide for enumerating hemophagocytes. Two hundred and fifty intact nucleated cells were counted in each quadrant on a single aspirate per case in areas with the highest density of hemophagocytes. Histiocytes were tallied by the lineage of ingested cells (mature RBCs, nRBCS, granulocytes, and lymphocytes) and the hematopoietic progenitor cell (HPC) sum was calculated as the total number of histiocytes containing ingested hematopoietic cells. The presence of multiple nucleated cells in a single hemophagocyte was also noted.

The methods used for the statistical analysis are described in the Online Supplementary Appendix.

\section{Results}

\section{Patients' characteristics}

Patients' characteristics of the 40 patients with HLH and 38 patients without HLH are summarized in Table 1. There were no significant differences in age or sex. HLH patients were more likely to present with underlying malignancy compared to the non-HLH group (56\% vs. $24 \% ; P<0.05)$, with diffuse large B-cell lymphoma (DLBCL) being the most common primary diagnosis among patients with HLH (Tables 1 and 2). Epstein-Barr virus (EBV) was the most common infectious trigger identified in patients with HLH; $32.5 \%$ of the patients in the HLH group had evidence of EBV infection by peripheral blood PCR detection or immunohistochemistry compared to $10.5 \%$ in the non-HLH group $(P<0.01)$.

As expected, patients diagnosed with HLH were more likely to present with clinical and laboratory findings satisfying each of the HLH-2004 diagnostic criteria (Table 1). Significant differences were observed between the HLH and non-HLH groups in the average value of each laboratory test (triglycerides, fibrinogen, ferritin, and soluble IL$2 \mathrm{r}$ ) and the number of patients satisfying each individual HLH-2004 criterion, with the exception of natural killer (NK)-cell function. Although impaired or absent NK-cell function testing is considered a valid screening tool for patients with genetic defects in cytotoxicity, this test is rarely ordered in adults with secondary HLH and has a high failure rate, limiting its diagnostic utility.

Hemophagocytosis was reported in the diagnostic bone 
marrow report of all 40 patients with $\mathrm{HLH}$, compared to 12 of $38(32 \%)$ in the non-HLH group $(P<0.01)$. Although evidence of hemophagocytosis may be reassuring for clinicians in making the diagnosis of HLH, hemophagocytosis is not required. In our cohort, the majority of patients ultimately diagnosed with HLH (73\%) fulfilled 5 or more criteria independently of evidence of hemophagocytosis in the bone marrow aspirate (Table 1). However, 9 patients (23\%) in the HLH group and 8 patients $(21 \%)$ in the non-HLH group met 4 criteria excluding hemophagocytosis, indicating that morphological assessment of the bone marrow aspirate was critical to the diagnosis in $22 \%$ of patients presenting with clinical features of concern for HLH.

Two patients in the HLH group satisfied fewer than 5 of the HLH-2004 criteria; however, both of these patients were surgically asplenic and the remainder of their clinical and laboratory findings, including the presence of hemophagocytosis on bone marrow aspirate, were compatible with the diagnosis. Four patients in the non-HLH group satisfied 5 criteria but were not diagnosed with HLH. The clinical notes of the treating physicians indicate that the chronicity of symptoms was considered to be inconsistent with HLH. None of these patients had hemophagocytosis identified in their bone marrow.

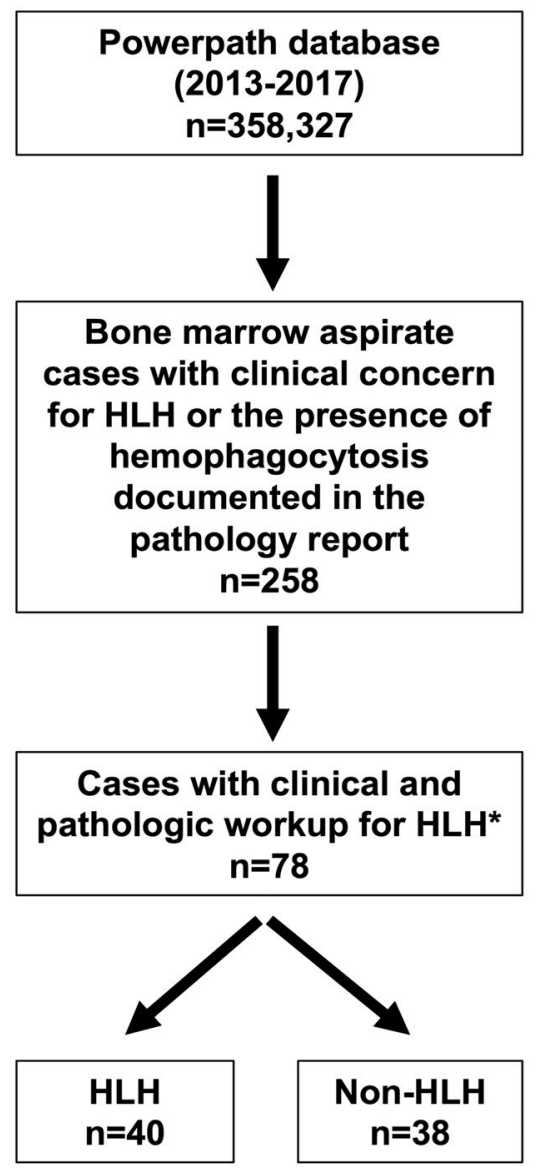

Figure 2. Flow chart for classification of patients. *Patients were excluded from the analysis if hemophagocytosis was incidentally noted independent of clinical concern for hemophagocytic lymphohistiocytosis $(\mathrm{HLH})$, slides were not available for review, HLH was considered but the diagnosis was equivocal after workup, or a documented history of HLH-directed treatment was noted prior to biopsy.
Developing predictive models for a morphological diagnosis of hemophagocytic lymphohistiocytosis

Patients diagnosed with HLH $(n=40)$ displayed significantly higher values of total hemophagocytes, and hemophagocytes with any of the cell lineages (RBCs, nRBCs, granulocytes, and lymphocytes) compared to non-HLH patients $(n=38)(P<0.001$ for all lineages) (Figures 1 and 3$)$. Correlation analysis demonstrated that each of the clinical and laboratory criteria included in the HLH-2004 criteria (excluding NK function) has a significant positive correlation with total number of hemophagocytes and hemophagocytes with each of the individual cell lineages, indicating that the degree of hemophagocytosis correlates with the diagnosis of HLH (Online Supplementary Figure S1).

Independently of one another, each lineage distinguished between HLH and non-HLH fairly well. Ingested $\mathrm{nRBCs}$ and ingested granulocytes had the highest area under the curve (AUC), indicating a high degree of correct classification of HLH and non-HLH subjects (AUC: 0.92), with threshold values of 2 ingested cells and 1 ingested cell, respectively. The sum of all four lineages also performed well at distinguishing between HLH and nonHLH with a threshold value of 6 (AUC: $0.92,95 \% \mathrm{CI}$ : $0.85,0.98)$.

Dichotomizing each lineage based on the threshold values described above and including all four in a decision tree, hemophagocytes ingesting granulocytes was chosen as the most important predictor of HLH, followed by nRBCs and lymphocytes (Figure 4A). Patients with an absence of hemophagocytes ingesting granulocytes had a $3 \%$ chance of having an HLH diagnosis. Patients with a presence of at least one hemophagocyte with an ingested granulocyte, two or more hemophagocytes with ingested $n R B C s$, and one hemophagocyte with ingested lymphocytes together were guaranteed to have an HLH diagnosis (100\% chance). Cross-validated (CV) AUC of this CART was 0.90 ; 95\% CI: 0.83-0.97.

Identifying lymphocytes within HPCs is rare, even in the presence of florid HLH. The highest number of HPCs containing lymphocytes was identified in a patient with

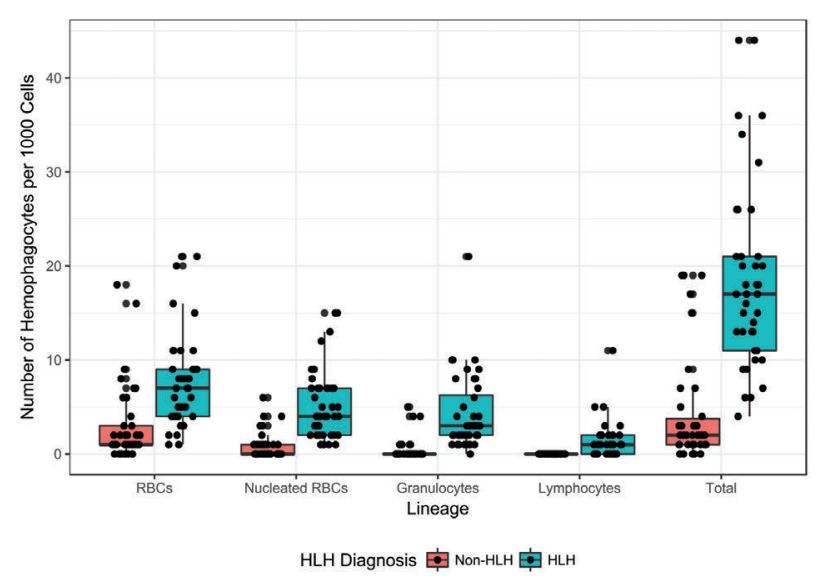

Figure 3. Values of hematopoietic progenitor cell (HPC) lineages and sum by hemophagocytic lymphohistiocytosis (HLH) diagnosis. Patients ultimately diagnosed as HLH had significantly higher values of all variables compared to nonHLH patients (Kruskal-Wallis rank sum test, $P<0.001$ ). 
DLBCL and based on morphology likely represented ingested tumor cells. Additionally, distinguishing between nucleated erythrocytes, lymphocytes, and hematopoietic progenitor cells within the cytoplasm of a histiocyte is challenging and subject to interpretive variability. As such, we created an additional CART excluding lymphocytes. This CART is identical to the first two levels of the previous model, with patients having a $92 \%$ chance of having an HLH diagnosis with a presence of at least one hemophagocyte with an ingested granulocyte and two or more hemophagocytes with ingested nRBCs (Figure 4B). While the CV AUC was identical to that of the first, overall accuracy was slightly higher in the CART without lymphocytes ( $88 \%$ CV accuracy vs. $86 \%$ ).

\section{Qualitative evaluation}

The initial description of virus-associated hemophagocytic syndrome described histiocytes that were "filled" with ingested hematopoietic elements. ${ }^{11}$ We frequently observe the phenomenon of multiple nucleated cells within individual histiocytes in patients with established HLH and hypothesized that this finding may be indicative of a pathological hemophagocytic state. To evaluate the significance of the degree of hemophagocytosis within individual hemophagocytes, we analyzed the presence of multiple nucleated cells within histiocytes (Figure 1). Our analysis ultimately demonstrated that at least one hemophagocyte-containing multiple nucleated cells was identified in 37 patients with HLH compared to only 4 patients in the non-HLH group (AUC: 0.91, 0.845-0.947), indicating that this binary qualitative feature performs similarly to the quantitative metrics described above for distinguishing patients with HLH from non-HLH patients (Table 3).

\section{Discussion}

Given the rarity of the diagnosis and non-specific clinical presentation, HLH is a challenging diagnosis for clinicians and pathologists. Once considered in the differential, expedient workup including evaluation of each of the HLH-2004 criteria is commonly pursued, as early therapeutic intervention improves outcomes in these often critically ill patients. Unfortunately, there is no defined threshold to satisfy the diagnostic criterion of hemophagocytosis in the bone marrow and evidence-based guidelines for reporting findings have not been established.

Towards this goal, we designed this retrospective study to determine whether quantitative features of hemophagocytosis at the time of initial bone marrow assessment are predictive of the ultimate diagnosis of $\mathrm{HLH}$ in patients presenting with clinical features concerning for the diagnosis. Given that HLH classically presents with multilineage cytopenia that is thought to result from consumption of hematopoietic cells by activated macrophages, ${ }^{12}$ we suspected that patients with HLH would be more likely to display hemophagocytosis of nucleated erythrocytes, granulocytes and lymphocytes compared to patients without HLH. In addition to evaluating for possible quantitative differences in hemophagocytosis, we simultaneously assessed for the presence of multiple nucleated cells within a single hemophagocyte as a candidate binary morphological feature that may differentiate patients with pathological hemophagocytosis.

Overall, we found that patients with HLH displayed significantly higher numbers of HPCs by each of the lineages examined (Table 3). We also identified quantitative thresh-

Table 2. Underlying medical conditions of patients in hemophagocytic lymphohistiocytosis (HLH) and non-HLH groups.

\begin{tabular}{|c|c|}
\hline $\begin{array}{l}\text { HLH } \\
\text { Diagnosis (N. of patients) }\end{array}$ & $\begin{array}{c}\text { Non-HLH } \\
\text { Diagnosis (N. of patients) }\end{array}$ \\
\hline Diffuse large B-cell lymphoma (9) & Classical Hodgkin lymphoma $\left(\mathrm{EBV}^{+}\right)(2)$ \\
\hline Peripheral T-cell lymphoma (6) & Diffuse Large B-cell lymphoma (1) \\
\hline NK/T-cell lymphoma/leukemia (2) & Peripheral T-cell lymphoma (2) \\
\hline EBV+ lymphoproliferative disorder (2) & T-Cell Acute lymphoblastic leukemia (1) \\
\hline Classical Hodgkin Lymphoma (EBV+) (1) & FIP1L1-PDGFRa myeloid leukemia (1) \\
\hline Langerhans cell histiocytosis (1) & Blastic plasmacytoid dendritic cell neoplasm (1) \\
\hline Primary effusion lymphoma (HIV+and HHV-8+) (1) & Autoimmune hemolytic anemia (2) \\
\hline Juvenile xanthogranuloma (1) & Systemic lupus erythematosus (5) \\
\hline B-cell acute lymphoblastic leukemia (1) & Adult onset still's disease (3) \\
\hline Rheumatoid arthritis (3) & Autoimmune hepatitis (1) \\
\hline Systemic lupus erythematosus (2) & Severe acquired neutropenia (1) \\
\hline Sarcoidosis (2) & Aplastic anemia (1) \\
\hline Dermatomyositis (1) & Autoimmune disorder NOS \\
\hline Atypical Kawasaki disease (1) & Dermatomyositis (1) \\
\hline Ulcerative colitis (1) & Autoimmune lymphoproliferative Syndrome (1) \\
\hline Idiopathic (5) & Mixed connective tissue disease (1) \\
\hline Post ventricular assist device (3) & Infection (6) \\
\hline Griscelli syndrome (homozygous Rab27a mutation) (1) & White cell aplasia (1) \\
\hline Homozygous perforin mutation (1) & Other (15) \\
\hline
\end{tabular}

N: number; NK: natural killer cell; EBV: Epstein-Barr virus; NOS: not otherwise specified. 
olds that can be used to accurately diagnose most cases of HLH: one granulocyte or two nucleated erythroid cells per 1000 nucleated cells. In addition, we utilized classification and regression tree analysis to identify the best combination of variables to create an even more specific and predictive model for discriminating patients with HLH. Finally, we showed that the presence of multiple nucleated cells within a single hemophagocyte was additionally predictive of a diagnosis of HLH among patients presenting with clinical findings of concern for the disease.

The initial description of virus-associated hemophagocytic syndrome (VAHS) described florid hemophagocytosis in 19 cases of immunosuppressed and previously healthy patients presenting with clinical features compatible with HLH.11 In 60 consecutive bone marrow aspirates used for comparison, they found erythrophagocytosis in 29 of 60 cases; however, the degree of phagocytosis was "never of a degree to be confused with the VAHS". This study conducted prior to the development of the HLH-2004 criteria provided initial evidence of the association of hemophagocytosis with VAHS (what we would now call HLH), and showed that the finding of erythrophagocytosis is frequently identified in bone marrow aspirates of patients without HLH, demonstrating a lack of specificity in isolation of clinical findings.

We conducted a similar non-blinded evaluation of 87 bone marrow aspirates from patients with de novo and post-treatment myeloid and lymphoid malignancies, benign cytopenias, and negative staging marrows, and identified predominantly erythrophagocytosis of mature RBCs in $39 \%$ of these cases. Applying the quantitative threshold determined from our analysis for engulfed non-nucleated RBCs (erythrophagocytes) to this dataset, we find only 3 of the 87 patients (5\%) would satisfy this morphological criterion (4 per 1000 cells). Similarly, if we apply the quantitative threshold for granulocytes (1 per 1000 cells), only 4 of the 87 patients (4\%) would satisfy this morphological criterion. Therefore, although we can indeed find rare examples of erythrophagocytosis in aspirate smears of a large minority of cases, the application of quantitative thresholds reveals a low incidence of clinically significant hemophagocytosis in a patient pop-

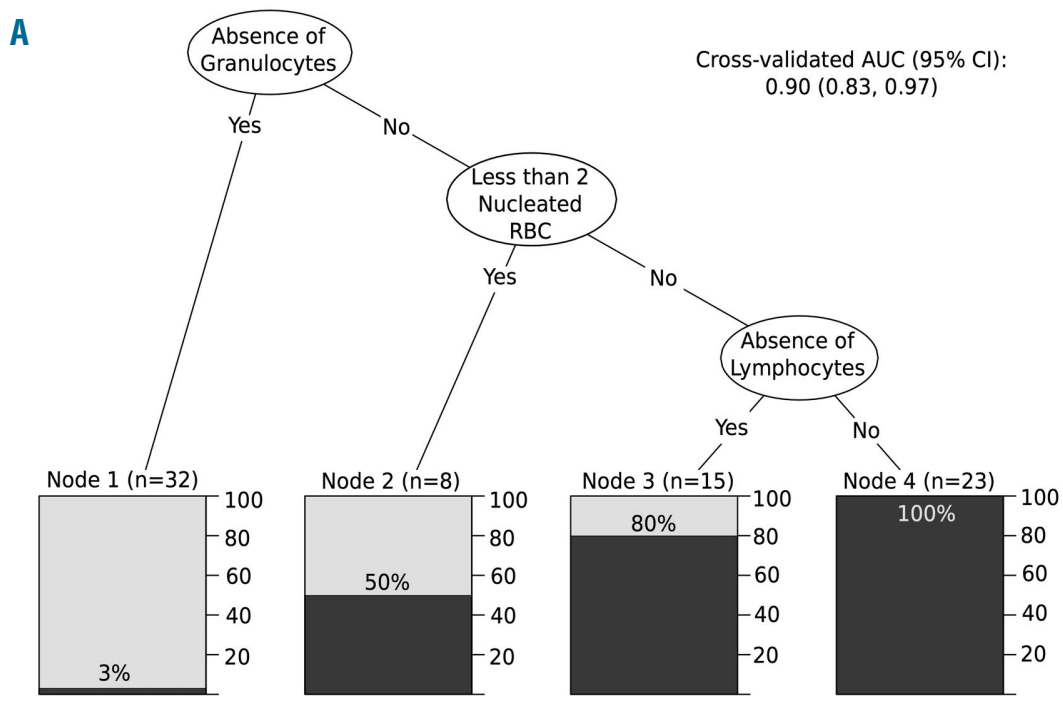

Percent Chance of HLH Diagnosis

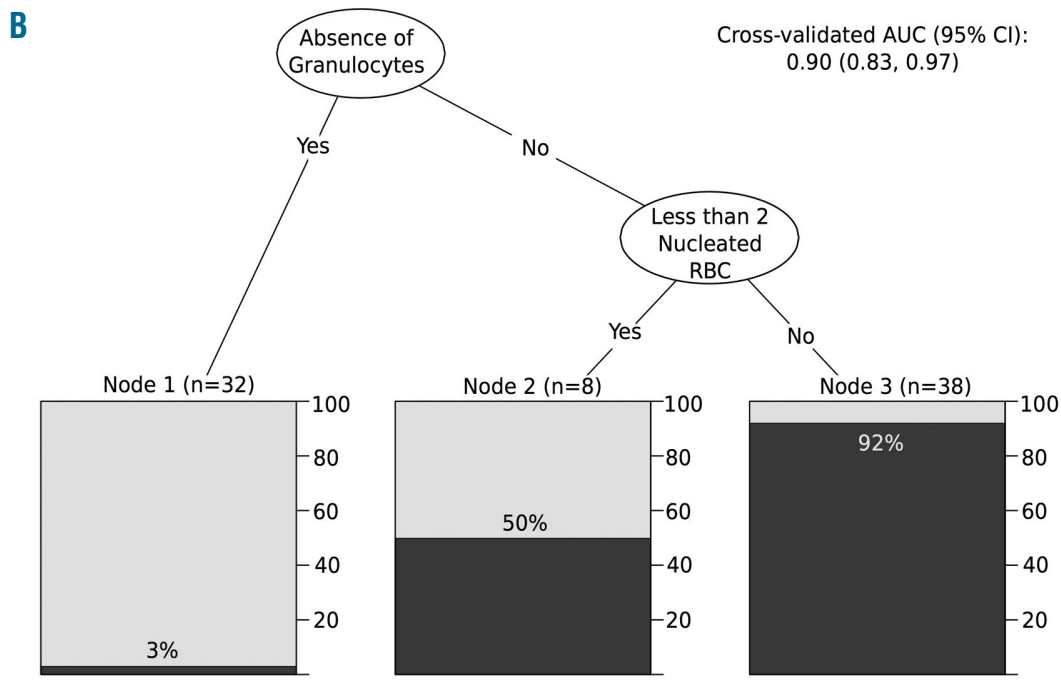

Percent Chance of HLH Diagnosis
Figure 4. Classification and regression trees (CART). Lineages were dichotomized based on the threshold values obtained from Table 3 and entered into the CART. Lineages are ordered in terms of relative importance to hemophagocytic lymphohistiocytosis $(\mathrm{HLH})$, where variables on the higher levels are deemed more important. The shaded area in each box corresponds to the probability of having an HLH diagnosis based on the path that leads to it. All four lineages were entered into the CART in (A), while lymphocytes were excluded from the CART in (B). 
Table 3. Quantities of hemophagocytic cells by lineage of ingested hematopoietic cells and optimal cutoff values derived from Youden's index.

\begin{tabular}{lcccc} 
Lineage & \multicolumn{2}{c}{ Mean (range)* } & Threshold & AUC (95\% Cl) \\
RBC & Non-HLH & $3.1(0-18)$ & 4 & $0.83(0.74-0.93)$ \\
nRBC & $7.7(1-21)$ & $0.8(0-6)$ & 2 & $0.92(0.87-0.98)$ \\
\hline Granulocyte & $5.2(1-15)$ & $0.5(0-5)$ & 1 & $0.92(0.86-0.99)$ \\
Lymphocyte & $4.3(0-21)$ & 0 & 1 & $0.80(0.72-0.88)$ \\
HPC sum & $1.3(0-11)$ & $4.3(0-19)$ & 6 & $0.92(0.85-0.98)$ \\
mHPC & $18.4(4-44)$ & 4 & NA & $0.91(0.85-0.95)$
\end{tabular}

RBC: red blood cells; nRBC: nucleated RBCs; HPC sum: total hematopoietic progenitor cell count from all four lineages; mHPC: multiple nucleated cells within a single HPC *Per 1000 cells.

ulation that reflects those seen in routine diagnostic practice.

A recent study by Ho et al. examined the specificity of hemophagocytosis for HLH by quantifying the absolute amount of hemophagocytosis identified in bone marrow aspirates of patients whose diagnostic pathology report described hemophagocytosis. ${ }^{10}$ They demonstrated that the presence of hemophagocytosis, even when present in a high amount, lacks specificity for HLH. Our institutional experience is consistent with their conclusion that significant hemophagocytosis is not predictive of the diagnosis of HLH in the absence of clinical features of concern for the disease; however, the presence of substantial hemophagocytosis is a relatively rare finding. Incidental hemophagocytosis was reported in 86 of 8097 (1.1\%) in-house bone marrow biopsy reports at Stanford University Hospital from 2013-2017. The majority of reports indicate "rare" hemophagocytosis $(61 \%)$ while the remainder describe "scattered", "occasional", or "brisk" hemophagocytosis. A subset $(n=12)$ of these latter cases was reviewed. All 12 cases demonstrated erythrophagocytosis. Half of the cases show ingested granulocytes and 3 cases demonstrated multiple nucleated cells within individual HPCs. None of these patients were ultimately diagnosed with HLH.

One major limitation of this study is its retrospective nature. Our population is limited to patients in whom the clinical diagnosis was thoroughly evaluated and a definitive determination was made concerning the diagnosis of
$\mathrm{HLH}$. We excluded patients in whom the ultimate diagnosis was ambiguous. Additionally, although our cohort includes all of the patients at our institution that met criteria for evaluation, including children, only a single patient in our cohort had a homozygous mutation diagnostic of primary $\mathrm{HLH}$, limiting the applicability of our findings to patients with genetic forms of the disease. Finally, we note that while CD107a testing has been described as a more sensitive test for primary $\mathrm{HLH},{ }^{13}$ this recently developed assay was not utilized in this retrospective cohort.

Ultimately, the diagnosis of HLH rests on the thorough assessment of patients in the appropriate clinical context. Similarly, microscopic examination of bone marrow aspirate smears in patients suspected of having $\mathrm{HLH}$ requires careful evaluation for the presence of hemophagocytosis. Our results demonstrate that quantitative thresholds of the lineage of ingested cells, either alone or in combination, accurately predict the eventual diagnosis of $\mathrm{HLH}$. We additionally demonstrate that identification of a single hemophagocyte containing multiple nucleated hematopoietic cells within its cytoplasm performs similarly to our quantitative approach. With external validation and further prospective study, we hope that these data will help to provide a method for pathologists and clinicians to systematically evaluate and accurately classify patients with HLH and contribute to establishment of consensus guidelines of diagnosis and reporting.

\section{References}

1. Jordan MB, Allen CE, Weitzman S, Filipovich AH, McClain KL. How I treat hemophagocytic lymphohistiocytosis. Blood. 2011;118(15):4041-4052.

2. George MR. Hemophagocytic lymphohistiocytosis: review of etiologies and management. J Blood Med. 2014;569-86.

3. Henter J-I, Horne A, Aricó M, et al. HLH2004: Diagnostic and therapeutic guidelines for hemophagocytic lymphohistiocytosis. Pediatr Blood Cancer. 2007;48(2):124131.

4. Buyse S, Teixeira L, Galicier L, et al. Critical care management of patients with hemophagocytic lymphohistiocytosis. Intensive Care Med. 2010;36(10):1695-1702.

5. Dhote R, Simon J, Papo T, et al. Reactive hemophagocytic syndrome in adult sys- temic disease: Report of twenty-six cases and literature review. Arthritis Care Res. 2003;49(5):633-639.

6. Machaczka M, Vaktnäs J, Klimkowska M Hägglund H. Malignancy-associated hemophagocytic lymphohistiocytosis in adults: a retrospective population-based analysis from a single center. Leuk Lymphoma. 2011;52(4):613-619.

7. Ramos-Casals M, Brito-Zerón P, LópezGuillermo A, Khamashta MA, Bosch X. Adult haemophagocytic syndrome. Lancet Lond Engl. 2014;383(9927):1503-1516.

8. Goel S, Polski JM, Imran H. Sensitivity and specificity of bone marrow hemophagocytosis in hemophagocytic lymphohistiocytosis. Ann Clin Lab Sci. 2012;42(1):21-25.

9. Glasser L LeGolvan M, Horwitz HM. Florid histiocytic hemophagocytosis following therapy with long acting G-CSF (pegfilgrastim). Am J Hematol. 2007;
82(8):753-757

10. Ho C, Yao X, Tian L, Li F-Y, Podoltsev N, $\mathrm{Xu}$ ML. Marrow assessment for hemophagocytic lymphohistiocytosis demonstrates poor correlation with disease probability. Am J Clin Pathol. 2014;141(1):6271.

11. Risdall RJ, McKenna RW, Nesbit ME, et al. Virus-associated hemophagocytic syndrome: a benign histiocytic proliferation distinct from malignant histiocytosis. Cancer. 1979;44(3):993-1002.

12. Zoller EE, Lykens JE, Terrell CE, et al. Hemophagocytosis causes a consumptive anemia of inflammation. J Exp Med. 2011; 208(6):1203-1214

13. Rubin TS, Zhang K, Gifford C, et al. Perforin and CD107a testing is superior to NK cell function testing for screening patients for genetic HLH. Blood. 2017; 129(22):2993-2999 